\title{
Classification and construction of six-quark basis states from parity eigenfunctions for $\mathrm{N}-\mathrm{N}$ processes
}

\author{
Fl. Stancu \\ Institute of Physics, B-5, University of Liege, B-4000 Liege, Belgium \\ and TRIUMF, Vancouver, British Columbia, Canada V6T $2 A 3$ \\ L. Wilets \\ Institute for Nuclear Theory, Department of Physics, University of Washington, Seattle, Washington 98195
}

(Received 24 February 1987)

\begin{abstract}
Six-quark configurations relevant to N-N scattering and bound states are constructed from parity eigenfunctions which can be obtained from any static independent particle model. The two lowest, one even and one odd, parity orbitals are considered. In the $(T S)=(10)$ and (01) sectors, there are 16 orthogonal color-singlet configurations of various orbital-spin-isospin symmetries; there are 7 in the $(00)$ and 25 in the (11) sectors. The results obtained here are compared with the cluster model approach for single particle $s$ states in each cluster. In that model, certain $p^{n} s^{6-n}$ components disappear from the wave function in the zero-separation limit, whereas the parity eigenfunction representation preserves a symmetry between even and odd parity states (with respect to the center of mass). Furthermore, cluster model configurations have been limited to three-quark clusters, which also limits the number of basis states. It is demonstrated here how parity eigenfunctions can be constructed from cluster model functions, and utilized to build the "missing" basis states.
\end{abstract}

\section{INTRODUCTION}

Six quark systems used for calculating the interaction energy in N-N scattering and bound states has been the subject of extensive study. A central problem has been the classification and construction of the basis states. On the one hand, the choice of a basis is arbitrary and irrelevant so long as sufficient states are included in the Hamiltonian diagonalization. On the other hand, one is limited by practical considerations to a finite set of states, and these should be chosen to be the most important ones.

We consider here two of the approaches which have been used in N-N studies: (1) the cluster model ${ }^{1-7}$ and (2) the independent particle model (IPM) or deformed shell model. ${ }^{8-10}$ Either can be used with the nonrelativistic, constituent quark model or with the various current quark models, including deformed MIT-type, chiral, or soliton bag models.

The cluster model has been employed in the sudden approximation. This means that the two nucleon clusters are not distorted as a function of inter-center separation except by the Pauli principle. We use $R$ and $L$ to designate the orbital part of right and left single-quark wave functions. They are centered at $\pm \frac{1}{2} Z$ ( $Z$ may be regarded as the separation coordinate in the cluster model or some deformation parameter for the IPM) with the same form (usually $s$ wave), so that there is only one function available. From this one function, states of even and odd parity can be generated.

Consider now IPM (or deformed shell model or molecular) wave functions. The single particle functions are derived from a static single-particle, axially and reflectionally symmetric model Hamiltonian $H_{0}$, such as can be obtained from constrained Hartree-Fock, MIT, or soliton mean field theories. Here the eigenstates of $H_{0}$, denoted by $\pi, m, f, c, n$ are classified by parity $\pi$, projection of angular momentum along the symmetry axis $m$, flavor $f(u$ or $d$ ), color $c$, and some other quantum number $n$ which may be, for example, the ordering of states of the same $\pi, m, f, c$ at $Z=0$. A highly schematic representation of the single particle eigenenergies is shown in Fig. 1. If only single particle dynamics is considered, three quantum numbers (not $m$ or $n$ ) are preserved in the collision process. (The Coriolis force mixes single particle states of different $m$.) This means that a (say) six quark state will conserve the amplitudes of the various components constructed from the product of six quark functions characterized by the set of conserved quantum numbers. Of course, there are quark-quark interactions $H^{\prime}$ due to the exchange of gluons or other fields which will lead to configuration mixing.

In the discussion which follows, we will limit ourselves to the lowest two orbitals, $R$ and $L$ for the cluster model, and one even and one odd parity state for the IPM. From these we will construct six quark states of various orbital, spin, and isospin symmetries all being color singlets. We will see that the number of such states with the same total spin-isospin quantum numbers is larger than previously. The additional states arise from clusters other than three body ones.

\section{A TWO QUARK EXAMPLE}

Consider the simple example of two quarks whose wave functions are characterized by $Z$. For both the cluster model and the IPM, one can define states which in the limit of large $Z$ may be written 


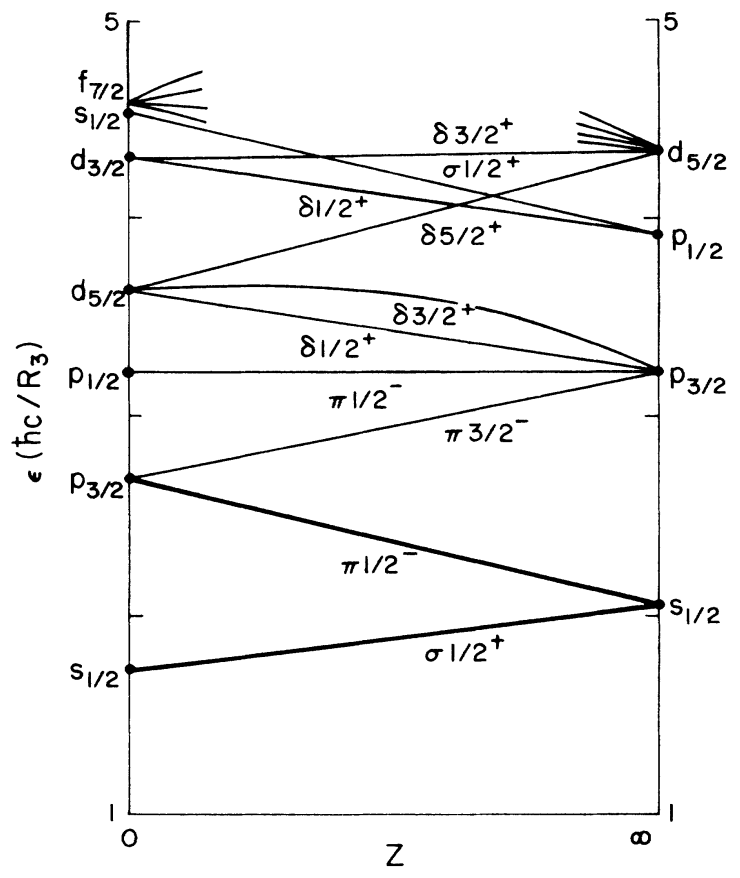

FIG. 1. Schematic representation of the eigenenergies of molecular-type orbitals for $0 \leq Z<\infty$. The $Z=\infty$ (separated bags) values are MIT eigenvalues in a bag of radius $R_{3}$; the $Z=0$ (united bag) values are for an MIT bag of radius $R_{6}=R_{3} / 2^{1 / 4}$. For intermediate $Z$, straight lines (except for one case) are used to connect the limiting values. The lines are labeled by $\left.|m|\right|^{\text {parity }}$; the levels are degenerate with respect to the sign of $m$. The Greek letter is to be identified with the Roman label at $\boldsymbol{Z}=0$, as introduced in Sec. II A. Note that each state at $\boldsymbol{Z}=\infty$ is degenerate with respect to parity as well as $m$. The heavy lines (lowest $\sigma$ and $\pi$ ) are the only states considered here.

$$
\begin{aligned}
& \psi\left(\mathbf{r}-\frac{1}{2} Z \hat{z}\right)=R, \\
& \psi\left(\mathbf{r}+\frac{1}{2} Z \hat{z}\right)=L,
\end{aligned}
$$

with $\langle R \mid R\rangle=\langle L \mid L\rangle=1$, and $\langle R \mid L\rangle \rightarrow 0$. These states have good parity about their centers $\pm \frac{1}{2} Z$, but not about their common center $\mathbf{r}=0$.

\section{A. The IPM}

The single particle Hamiltonian $H_{0}$ yields eigenstates which have good parity with respect to the common center. We label the parity states by $\sigma$ for even parity since it becomes an $s$ state as $Z \rightarrow 0$, and by $\pi$ for odd parity since it becomes a $p$ state as $Z \rightarrow 0$. (This can be generalized to $\sigma, \pi, \delta, \phi, \gamma, \ldots$ for states which become $s, p, d, f, g, \ldots)$; refer to Fig. 1 . Then asymptotically

$\left[\begin{array}{l}\sigma \\ \pi\end{array}\right] \rightarrow 2^{-1 / 2}(R \pm L), \quad 2^{-1 / 2}(\sigma \pm \pi) \rightarrow\left(\begin{array}{l}R \\ L\end{array}\right)$ as $Z \rightarrow \infty$.

By definition, $\langle\sigma \mid \sigma\rangle=\langle\pi \mid \pi\rangle=1$ and $\langle\sigma \mid \pi\rangle=0$ at any $Z$.
One can write spatially symmetric or antisymmetric two quark states which asymptotically have one quark about each center (one in each bag), so that

$$
\left(\begin{array}{l}
S \\
A
\end{array}\right) \rightarrow 2^{-1 / 2}[R(1) L(2) \pm L(1) R(2)] \text { as } Z \rightarrow \infty \text {. }
$$

The definition of $R$ and $L$ states has meaning only for $Z \rightarrow \infty$, but we can introduce pseudo-right and -left states $r$ and $l$,

$$
\left[\begin{array}{l}
r \\
l
\end{array}\right] \equiv 2^{-1 / 2}(\sigma \pm \pi) \text { for all } Z
$$

where $\langle r \mid r\rangle=\langle l \mid l\rangle=1$ and $\langle r \mid l\rangle=0$.

Then for all $Z$, one can write

$$
\begin{aligned}
& S=2^{-1 / 2}[\sigma(1) \sigma(2)-\pi(1) \pi(2)], \\
& A=-2^{-1 / 2}[\sigma(1) \pi(2)-\pi(1) \sigma(2)] .
\end{aligned}
$$

Note that the symmetric state has even total parity and the antisymmetric state has total odd parity. As $Z \rightarrow 0$, we find $S \rightarrow 2^{-1 / 2}\left(s^{2}-p^{2}\right)$ and $A \rightarrow-2^{-1 / 2}(s p-p s)$. (In this shorthand notation, the particle coordinate ordering is 1,2 .) In both cases there is equal probability of finding one $s$ and one $p$ quark at $Z=0$.

\section{B. The cluster model}

In the cluster model the states $R$ and $L$ are defined for all $Z$ by the form (2.1). They are not orthogonal except at $Z=\infty$; at $Z=0$ one has $\langle R \mid L\rangle=1$. One can construct good parity, orthonormal states for all $Z$ by setting

$$
\left(\begin{array}{l}
\sigma \\
\pi
\end{array}\right) \equiv[2(1 \pm\langle R \mid L\rangle)]^{-1 / 2}(R \pm L)
$$

Reexpressing the $R$ and $L$ states in terms of the parity eigenstates, we find

$$
\left(\begin{array}{l}
R \\
L
\end{array}\right)=\left(\frac{1+\langle R \mid L\rangle}{2}\right)^{1 / 2} \sigma \pm\left(\frac{1-\langle R \mid L\rangle}{2}\right)^{1 / 2} \pi .
$$

Note that the odd parity component of either $R$ or $L$ vanishes in the limit $Z \rightarrow 0$.

Consider now the symmetrized and normalized product of a two quark $R L$ state,

$$
\left[\begin{array}{l}
S \\
A
\end{array}\right]=\left[2\left(1 \pm\langle R \mid L\rangle^{2}\right)\right]^{-1 / 2}[R(1) L(2) \pm L(1) R(2)]
$$

or

$$
\begin{aligned}
& S=\left[2\left(1+\langle R \mid L\rangle^{2}\right)\right]^{-1 / 2}[(1+\langle R \mid L\rangle) \sigma(1) \sigma(2) \\
&-(1-\langle R \mid L\rangle) \pi(1) \pi(2)], \\
& A=-\frac{1}{\sqrt{2}}[\sigma(1) \pi(2)-\pi(1) \sigma(2)] .
\end{aligned}
$$

Again we see that the symmetric state is of even parity and the antisymmetric state has odd parity. The $\sigma \pi$ structure of the $A$ states is independent of $Z$, as must be the case. The relative $\sigma \sigma$ and $\pi \pi$ components of the $S$ states vary with $Z$ and as $Z \rightarrow 0$ the $\pi^{2} \rightarrow p^{2}$ component 
vanishes. This is an essential difference between the cluster and IPM models.

Nevertheless, even within the framework of the cluster model, one can write two-particle states of the form (2.5). By utilizing the parity eigenstates (2.6) in definition (2.4), one has

$$
\begin{aligned}
\left(\begin{array}{l}
r \\
l
\end{array}\right) & =2^{-1 / 2}(\sigma \pm \pi) \\
& =\frac{1}{2}\left[\frac{R+L}{(1+\langle R \mid L\rangle)^{1 / 2}} \pm \frac{R-L}{1-\langle R \mid L\rangle)^{1 / 2}}\right] .
\end{aligned}
$$

Clearly $r \rightarrow R$ and $l \rightarrow L$ as $Z \rightarrow \infty$. We can introduce the two-body symmetric state, $\widetilde{S}$ and antisymmetric state, $\widetilde{A}$,

$$
\left[\begin{array}{c}
\tilde{S} \\
\tilde{A}
\end{array}\right]=2^{-1 / 2}[r(1) l(2) \pm l(1) r(2)],
$$

which expressed in terms of parity eigenstates $\sigma$ and $\pi$ have exactly the same form as (2.5). The $\widetilde{A}$ and $A$ states are identical to one another, but the $\widetilde{S}$ and $S$ states are quite distinct. In the limit $Z \rightarrow 0$,

$$
\begin{aligned}
& \tilde{S} \rightarrow 2^{-1 / 2}\left(s^{2}-p^{2}\right), \\
& S \rightarrow S^{2} .
\end{aligned}
$$

The disappearance of the $p$ state as $Z \rightarrow 0$ in the cluster model is an artifact of the model wave function used. The problem recurs in the $\mathrm{N}-\mathrm{N}$ wave function, as will be seen in Sec. III.

It is of interest to compare the $R, L$ with the $r, l$ functions. For this purpose we use Gaussian functions

$$
\left(\begin{array}{l}
R \\
L
\end{array}\right)=\pi^{-3 / 4} e^{-(\mathbf{r} \mp Z \hat{z} / 2)^{2} / 2} .
$$

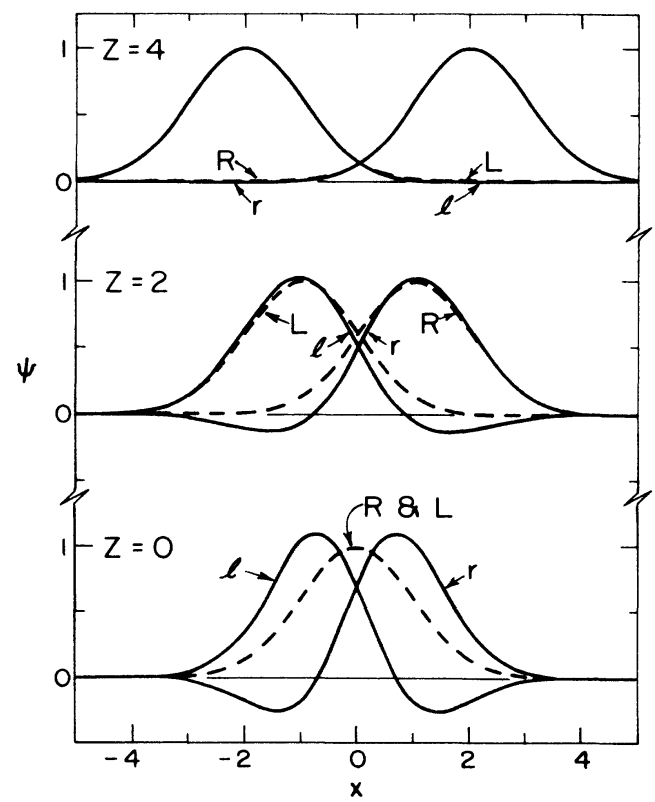

FIG. 2. Functions $R, L, r$, and $l$ along the symmetry axis for $Z=0,2,4$, using the Gaussian form (2.12).

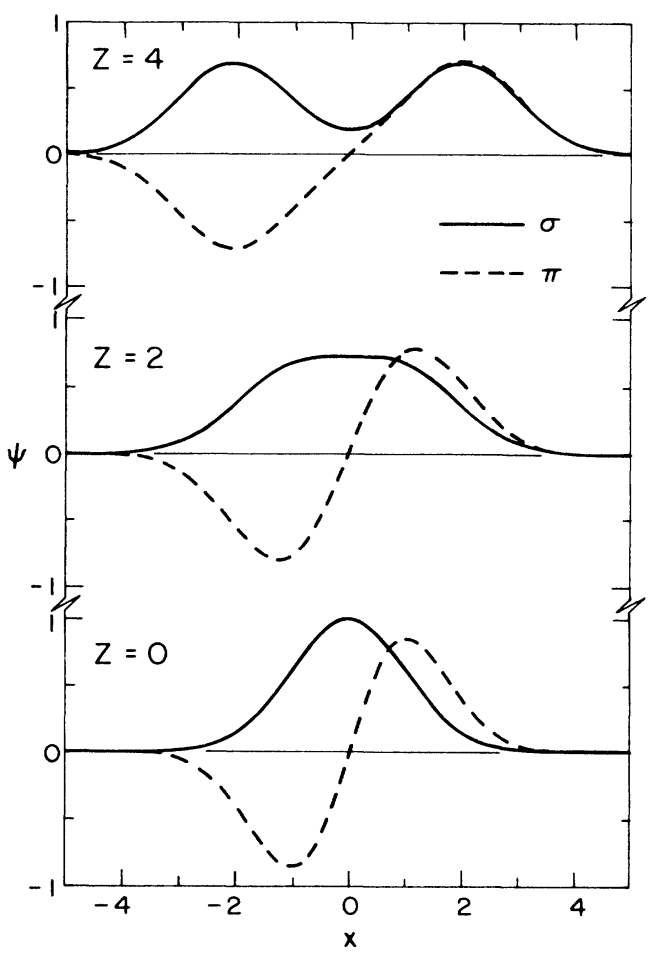

FIG. 3. Same as Fig. 1 but for $\sigma$ and $\pi$.

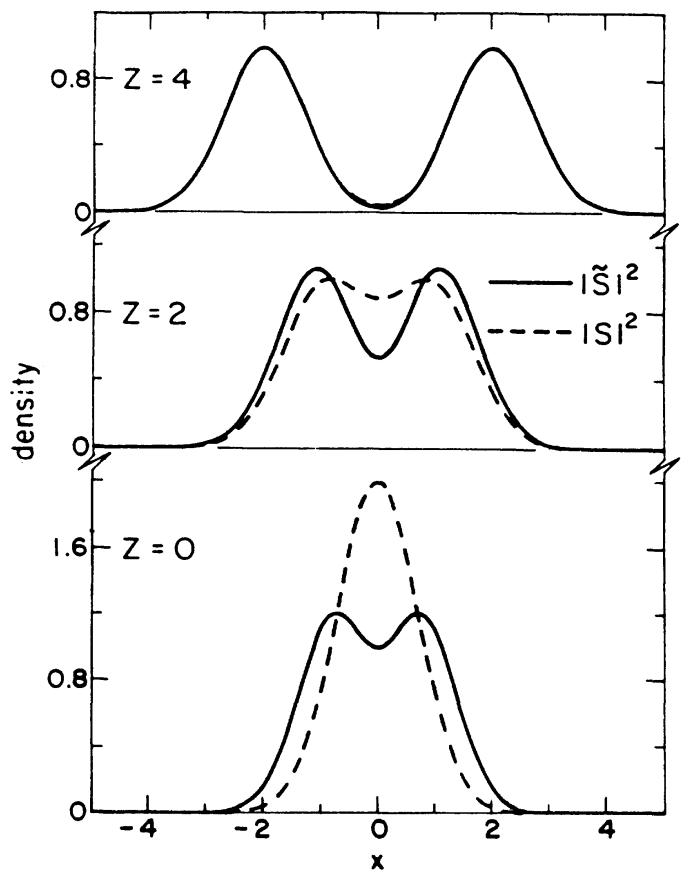

FIG. 4. Same as Fig. 1 for the densities of the two body symmetric states $|S|^{2}$ [cf. Eq. (2.9a)] and $|\widetilde{S}|^{2}$ [cf. Eq. (2.11)]. 
In Fig. 2 are plotted $R, L, r$, and $l$ along the symmetry axis. In Fig. 3 are plotted $\sigma$ and $\pi$. In Fig. 4 , the densities $|S|^{2}$ and $|\widetilde{S}|^{2}$ are plotted. In all cases, $Z$, is taken to be 0,2 , and 4 .

\section{SIX QUARK STATES}

In this section we describe the structure of six-quark states built from the orthogonal states $r$ and $l$ introduced previously.

The orbital, spin, flavor, and color degrees of freedom are taken into account through the classification scheme introduced by Harvey. Accordingly the orbital part of the Young symmetry $[f]$ is coupled to a color-isospin-spin part of dual symmetry $[\widetilde{f}]$ to give a totally antisymmetric wave function. For simplicity we also assume that the lowest state of a baryon has orbital symmetry $[f]=[3]$, hence the only possible symmetries for six-quark states are [6], [51], [42], and [33]. In a relativistic model description of the baryon, the quarks occupy $j=\frac{1}{2}$ ( $s$ wave) states. Therefore the symmetry group associated with these states is $\mathbf{S U}(2)$, like in the nonrelativistic case. In both cases, we shall refer to it as $\mathrm{SU}_{S}(2)$. The colorisospin-spin space, classified as $\mathrm{SU}_{T}(2) \times \mathrm{SU}_{S}(2)$ states of isospin $T$ and spin $S$, and the $\mathrm{SU}_{c}(3)$ state of color symmetry [222] are taken from Table I of Ref. 1.

Our states are different from those used by Harvey ${ }^{1-3}$ through the single-particle content of the orbital functions $\psi_{6}(f Y)$ where $Y$ stands for the Yamanouchi label of the Young tableau $f$. The cluster states which we denote by $R$ and $L$ correspond, in Harvey's notation, to $s_{+}$and $s_{-}$. In the construction of six quark wave func- tions we substitute the orthonormal $r$ and $l$ for $s_{+}$and $s_{-}$. These $r$ and $l$, however, can be obtained either from a cluster model or from an IPM, as discussed in the preceding section. They ensure the orthonormality of the $\psi_{6}(f Y)$ functions at all separation distances $Z$. This property simplifies the structure of the wave functions. The use of $r$ and $l$ states also gives rise to a richer single particle composition of the six-quark states as compared to the cluster states $R$ and $L$. To better understand this difference let us consider, as an example, the wave function $\psi_{6}([42])$ represented by the Young tableau

\begin{tabular}{|l|l|l|l|}
\hline 1 & 3 & 5 & 6 \\
\hline 2 & 4 & \multicolumn{2}{|c}{} \\
\cline { 1 - 1 } & &
\end{tabular}

and having the configuration $r^{3} l^{3}$. Its normalized expression reads

$\psi_{6}([42])=\frac{1}{2 \sqrt{2}}\left(r_{1} l_{2}-l_{1} r_{2}\right)\left(r_{3} l_{4}-l_{3} r_{4}\right)\left(r_{5} l_{6}+l_{5} r_{6}\right)$,

where the subscripts refer to the particle labels. In terms of $\sigma$ and $\pi$ we have

$$
\begin{array}{r}
\psi_{6}(42)=\frac{1}{2 \sqrt{2}}[(\pi \sigma \pi \sigma \sigma \sigma-\sigma \pi \pi \sigma \sigma \sigma-\pi \sigma \sigma \pi \sigma \sigma \\
+\sigma \pi \sigma \pi \sigma \sigma)-(\sigma \pi \sigma \pi \pi \pi-\pi \sigma \sigma \pi \pi \pi \\
-\sigma \pi \pi \sigma \pi \pi+\pi \sigma \pi \sigma \pi \pi)] .
\end{array}
$$

\begin{tabular}{|c|c|c|c|}
\hline Name & $r^{n} l^{6-n}$ configuration & & $\sigma^{n} \pi^{6-n}$ configuration \\
\hline \\
\hline $60^{+}[6]$ & $\frac{1}{\sqrt{2}}\left[\left(r^{6}[6]\right)+\left(l^{6}[6]\right)\right]$ & +2 & $\frac{1}{4 \sqrt{2}}\left[\left(\sigma^{6}[6]\right)+\left(\pi^{6}[6]\right)+\sqrt{15}\left(\sigma^{4} \pi^{2}[6]\right)+\sqrt{15}\left(\sigma^{2} \pi^{4}[6]\right)\right.$ \\
\hline $51+[6]$ & $\frac{1}{\sqrt{2}}\left[\left(r^{5} l[6]\right)+\left(r l^{5}[6]\right)\right]$ & & $\frac{1}{4}\left[\sqrt{3}\left(\sigma^{6}[6]\right)-\sqrt{3}\left(\pi^{6}[6]\right)+\sqrt{5}\left(\sigma^{4} \pi^{2}[6]\right)-\sqrt{5}\left(\sigma^{2} \pi^{4}[6]\right)\right]$ \\
\hline $42^{+}[6]$ & $\frac{1}{\sqrt{2}}\left[\left(r^{4} l^{2}[6]\right)+\left(r^{2} l^{4}[6]\right)\right]$ & & $\frac{1}{4 \sqrt{2}}\left[\sqrt{15}\left(\sigma^{6}[6]\right)+\sqrt{15}\left(\pi^{6}[6]\right)-\left(\sigma^{4} \pi^{2}[6]\right)-\left(\sigma^{2} \pi^{4}[6]\right)\right]$ \\
\hline $33[6]$ & $\left(r^{3} l^{3}[6]\right)$ & & $\frac{1}{4}\left[\sqrt{5}\left(\sigma^{6}[6]\right)-\sqrt{5}\left(\pi^{6}[6]\right)-\sqrt{3}\left(\sigma^{4} \pi^{2}[6]\right)+\sqrt{3}\left(\sigma^{2} \pi^{4}[6]\right)\right]$ \\
\hline $51+[51]$ & $\frac{1}{\sqrt{2}}\left[\left(r^{5} l[51]\right)+\left(r l^{5}[51]\right)\right]$ & & $-\frac{1}{\sqrt{2}}\left[\left(\sigma^{4} \pi^{2}[51]\right)-\left(\sigma^{2} \pi^{4}[51]\right)\right]$ \\
\hline $42^{+}[51]$ & $\frac{1}{\sqrt{2}}\left[\left(r^{4} l^{2}[51]\right)+\left(r^{2} l^{4}[51]\right)\right]$ & & $-\frac{1}{\sqrt{2}}\left[\left(\sigma^{4} \pi^{2}[51]\right)+\left(\sigma^{2} \pi^{4}[51]\right)\right]$ \\
\hline $42+[42]$ & $\frac{1}{\sqrt{2}}\left[\left(r^{4} l^{2}[42]\right)+\left(r^{2} l^{r 4}[42]\right)\right]$ & & $\frac{1}{\sqrt{2}}\left[\left(\sigma^{4} \pi^{2}[42]\right)+\left(\sigma^{2} \pi^{4}[42]\right)\right]$ \\
\hline 3342 & $\left(r^{3} l^{3}[42]\right)$ & & $\frac{1}{\sqrt{2}}\left[\left(\sigma^{4} \pi^{2}[42]-\left(\sigma^{2} \pi^{4}[42]\right)\right]\right.$ \\
\hline \multicolumn{4}{|c|}{ Odd parity } \\
\hline $51^{-}[51]$ & $\frac{1}{\sqrt{2}}\left[\left(r^{5} l[51]\right)-\left(r l^{5}[51]\right)\right]$ & & $\left.-\frac{1}{4} \sqrt{2}\left(\sigma^{5} \pi[51]\right)-\sqrt{2}\left(\sigma \pi^{5}[51]\right)+2 \sqrt{3}\left(\sigma^{3} \pi^{3}[51]\right)\right]$ \\
\hline $42^{-}[51]$ & $\frac{1}{\sqrt{2}}\left[\left(r^{4} l^{2}[51]\right)-\left(r^{2} l^{4}[51]\right)\right]$ & & $-\frac{1}{\sqrt{2}}\left[\left(\sigma^{5} \pi[51]\right)+\left(\sigma \pi^{5}[51]\right)\right]$ \\
\hline $33[51]$ & $\left(r^{3} l^{3}[51]\right)$ & & $-\frac{1}{4}\left[\sqrt{6}\left(\sigma^{5} \pi[51]\right)-\sqrt{6}\left(\sigma \pi^{5}[51]\right)-2\left(\sigma^{3} \pi^{3}[51]\right)\right]$ \\
\hline $42^{-}[42]$ & $\frac{1}{\sqrt{2}}\left[\left(r^{4} l^{2}[42]\right)-\left(r^{2} l^{4}[42]\right)\right]$ & & $\left(\sigma^{3} \pi^{3}[42]\right)$ \\
\hline $33[33]$ & $\left(r^{3} l^{3}[33]\right)$ & & $-\left(\sigma^{3} \pi^{3}[33]\right)$ \\
\hline
\end{tabular}

Here the particle labels are understood to be in the order

TABLE I. Transformation from the $r-l$ to the $\sigma-\pi$ representations. 
$1,2, \ldots, 6$. We can see that in the limit $Z \rightarrow 0$ this expression contains the configuration $s^{2} p^{4}$ in addition to the $s^{4} p^{2}$ obtained by Harvey. In general, we find that with the replacement of $R$ and $L$ by $r$ and $l$ states any configuration which in the spherical limit contained $n s$ states and $6-n p$ states now appears in combination with a configuration with $s$ and $p$ interchanged. For higher symmetries as [6] or [51] extra configurations appear. Our results are summarized in Table I. In this table we list the even and odd parity normalized six quark states formed from all possible configurations $r^{n} l^{6-n}$ with $n=1,2, \ldots, 6$ and their corresponding $\sigma^{n} \pi^{(6-n)}$ structure. One can see that a given structure can be reached not only through occupying the $r$ and $l$ states with three quarks each but also from clustering the quarks, two in one bag, four in the other bag, etc. The limiting structure $(Z \rightarrow 0)$ of each symmetry state can automatically be obtained by the replacement of $\sigma$ by $s$ and of $\pi$ by $p$.

Based on the orbital wave functions of Table I we can now build color singlet totally antisymmetric states. For this purpose we use Table I of Ref. 1 where are listed the SU(4) symmetries required by each orbital symmetry. Table II gives these combined symmetries for the $(T=0$, $S=1),(T=1, S=0),(T=0, S=0)$, and $(T=1, S=1)$ sectors. The orbital symmetry (column 2 ) is specified with the notation of Table I. The multiplicities of a $(T S)$ sector within a given $\mathrm{SU}(4)$ representation are indicated (column 3) whenever larger than one. There are 16 channels in the $(T=0, S=1)$ or $(T=1, S=0)$ sectors, seven in the $(T=0, S=0)$ sector, and 25 in the $(T=1$, $S=1)$ sector.

In the manner of Table II of Ref. 1, the 16 even total parity states can be transformed into 16 channels

TABLE II. The orbital and SU(4) content for the colorsinglet six quark states in the sectors $(T S)=(01),(10),(00)$, and (11).

\begin{tabular}{|c|c|c|}
\hline Sector & Orbital & SU(4) \\
\hline $\begin{array}{l}T=0, S=1 \\
T=1, S=0\end{array}$ & $\begin{array}{c}60^{+}[6], 51^{+}[6], 42^{+}[6], 33[6] \\
51^{+}[51], 42^{+}[51] \\
42^{+}[42], 33[42]\end{array}$ & $\begin{array}{c}\{33\} \\
\{321\} \\
\{51\} \\
\{33\} \\
\{411\} \\
\{2211\} \\
\{321\}\end{array}$ \\
\hline$T=0, S=0$ & $\begin{array}{c}51^{-}[51], 42^{-}[51], 33[51] \\
33[33]\end{array}$ & $\begin{array}{c}\{42\} \\
\{6\} \\
\{42\} \\
\{222\} \\
\{3111\}\end{array}$ \\
\hline$T=1, S=1$ & $\begin{array}{c}51^{-}[51], 42^{-}[51], 33[51] \\
42^{-}[42], 33[42]\end{array}$ & $\begin{array}{c}\{42\} \times 2 \\
\{321\} \times 2 \\
\{51\} \\
\{411\} \\
\{321\} \times 2 \\
\{6\} \\
\{42\} \times 2 \\
\{222\} \\
\{311\} \\
\end{array}$ \\
\hline
\end{tabular}

classified asymptotically as follows: one physical NN state with three quarks per center (or cluster or bag),

one physical $\Delta \Delta$ state with three quarks per center, four "hidden color" states with three quarks per center,

seven states of the form $R^{2} L^{4}$ or $R^{4} L^{2}$,

two states of the form $R L^{5}$ or $R^{5} L$,

one state of the form $L^{6}$ or $R^{6}$.

At this point an important remark is in order. Using Table I we can see that Harvey's N-N state at $Z=0$ goes over to $s^{6}+s^{4} p^{2}$ while our N-N state has the extra limiting configurations $p^{6}+p^{4} s^{2}$. We already saw an example of this in the two-quark case of Sec. II. In the cluster model, $Z \rightarrow 0$ must be taken as a careful limit because all $p$ states vanish and a normalization factor in the denominator saves the $s^{4} p^{2}$ configuration. The vanishing of the $p$ states is an artifact of the cluster model. A similar analysis can be done for the odd total parity (00) and (11) sectors.

Even by retaining only $\mathrm{SU}(4)$ symmetries associated with pairs of nucleons and deltas (asterisked symmetries of Ref. 1) the present space remains larger than in previous studies. This is due to the presence of configurations with 6-0, 5-1, 4-2 quarks per center. It is evident that only physical states have finite energies for separated structures, but the other channels, which do not have three quarks per structure, can play an important role for small $Z$, as do the hidden color states. The $r^{6}+l^{6}$ configuration requires special consideration. Asymptotically, it corresponds to displaced six-quark structures of finite energy, and is spurious.

\section{SUMMARY AND CONCLUSIONS}

Six quark configurations relevant for $\mathrm{N}-\mathrm{N}$ processes are constructed from single-particle parity and $m= \pm \frac{1}{2}$ eigenfunctions, where $m$ is the projection of the total angular momentum along the symmetry axis. In the independent particle model such functions are eigenfunctions of a one-body Hamiltonian which can be constructed in the constrained Hartree-Fock or mean field approximation, or deformed bag model, etc. Such a static Hamiltonian is assumed to possess reflectional and axial symmetry. In analogy with (homopolar) diatomic collisions or molecules, these states can also be referred to as molecular orbitals. We restrict ourselves to the lowest even $(\sigma)$ and odd $(\pi)$ functions. As a function of the separation coordinate or deformation parameter, $Z$, we have $\sigma \rightarrow s_{1 / 2}$ and $\pi \rightarrow p_{3 / 2}$ as $Z \rightarrow 0$; for large $Z$ we find $2^{-1 / 2}(\sigma \pm \pi) \rightarrow R, L$ where $R$ and $L$ are $s$-state functions about their respective centers. We have specifically considered even total parity configurations [ $L$ even, $(T S)=(01)$ and (10)] and odd total parity $[L$ odd, $(T S)=(00)$ and (11)] N-N process.

The structure here is contrasted with the cluster model structure. Using the IPM functions provides a larger space. One can, however, use the cluster model functions to construct one-body parity eigenfunctions and use these to generate IPM-type configurations.

"Potentials" obtained in nonrelativistic cluster models are upper bounds. Inclusion of more configurations and 
distortion will lower the energy at all separations. Potentials, however, do not contain sufficient information to solve for physical quantities. One must solve the dynamic equations in order to obtain phase shifts or binding energies. This has been done for the nonrelativistic cluster model (e.g., Ref. 5) and for the relativistic soliton bag model (e.g., Ref. 10). In both cases, one obtains the effects of a repulsive core even if the static "potential" does not exhibit it.

In a subsequent paper, we shall present the results of channel coupling through one-gluon exchange for $Z=0$ based on the IPM configurations constructed here, and compare these with the results of the more restricted cluster configuration.

\section{ACKNOWLEDGMENTS}

This work was supported in part by the U.S. Department of Energy. One of us (F.S.) wishes to thank the Institute for Nuclear Theory at the University of Washington and the Theory Group at TRIUMF for their kind hospitality, and to acknowledge support from the Belgian Fond National de la Recherche Scientifique.
${ }^{1}$ M. Harvey, Nucl. Phys. A352, 301 (1981).

${ }^{2}$ M. Harvey, Nucl. Phys. A352, 326 (1981).

${ }^{3}$ M. Harvey, J. Letourneux, and B. Lorazo, Nucl. Phys. A424, 428 (1984).

${ }^{4}$ M. Oka and K. Yazaki, Quarks in Nuclei, edited by W. Weise (World Scientific, Singapore, 1984), p. 489, and references therein.

${ }^{5}$ A. Faessler, F. Fernandez, G. Lübeck, and K. Shimizu, Nucl. Phys. A402, 555 (1983).
${ }^{6}$ K. Maltman and N. Isgur, Phys. Rev. D 29, 952 (1984).

${ }^{7}$ Y. Suzuki and K. T. Hecht, Nucl. Phys. A420, 525 (1984); A446, 625 (1986).

${ }^{8}$ C. DeTar, Phys. Rev. D 17, 302 (1978); 17, 323 (1978).

${ }^{9}$ A. Schuh, Doctoral Dissertation, University of Heidelberg, 1985.

${ }^{10}$ A. Schuh, H. J. Pirner, and L. Wilets, Phys. Lett. 174B, 10 (1986). 